\title{
SOME DECIDABLE DIOPHANTINE PROBLEMS: POSITIVE SOLUTION TO A PROBLEM OF DAVIS, MATIJASEVIČ AND ROBINSON ${ }^{1}$
}

\author{
MOSHE KOPPEL
}

\begin{abstract}
An algorithm is given for determining whether or not a finite system of conditions of the types $a \mid B, a<B, a$ is a square, possess a simultaneous solution in positive integers. Various generalizations are also obtained.
\end{abstract}

In 1970, Matijasevič proved that there is no algorithm to determine if a general polynomial Diophantine equation has a solution in positive integers (see, for example, Davis, Matijasevič and Robinson [1]). A particularly neat formulation of this theorem can be obtained from the observation made by Skolem [6] that any Diophantine equation can be reduced to a system of conditions of types $\alpha+\beta=\gamma, \alpha \cdot \beta=\gamma$. The theorem then reads: There is no algorithm to determine whether a system of conditions of types: $\alpha+\beta=$ $\gamma, \alpha \cdot \beta=\gamma$ has a solution in positive integers.

There are other relations such that certain systems of conditions using those relations are equivalent to $\alpha+\beta=\gamma$ and $\alpha \cdot \beta=\gamma$. For example, consider the relations $\alpha+1=\beta, \alpha \cdot \beta=\gamma$. Then, $x+y=z$ is equivalent to the system $x+1=\alpha_{1}, z+1=\alpha_{2}, \alpha_{1} \cdot \alpha_{2}=\alpha_{3}, \alpha_{3}+1=\alpha_{4}, \alpha_{2} \cdot y=\alpha_{5}$, $\alpha_{5}+1=\alpha_{6}, \alpha_{6} \cdot \alpha_{4}=\alpha_{7}, \alpha_{1} \cdot y=\alpha_{8}, \alpha_{8}+1=\alpha_{9}, \alpha_{9} \cdot \alpha_{2}=\alpha_{10}, \alpha_{10} \cdot \alpha_{2}=$ $\alpha_{11}, \alpha_{11}+1=\alpha_{7}$. (This is simply an expansion of $s(s x \cdot s z) \cdot s(y \cdot s z)=s(s z$. $s z \cdot s(s x \cdot y)$ ) where $s \alpha=\alpha+1$.) Consequently, there is no algorithm to determine if a system of conditions of types: $\alpha+1=\beta, \alpha \cdot \beta=\gamma$ has a solution in positive integers.

Similar methods have been used to extend the theorem to various classes of relations (see, for example, Robinson [4] and Schwartz [5]). In particular, Kosovskii [3] showed that there is no algorithm to determine if a system of conditions of types: $\alpha+\beta=\gamma, \alpha \mid \beta, \alpha=\square$ has a solution in positive integers. ( $\alpha=\square$ means that $\alpha$ is a perfect square.)

This result motivated the problem posed by Davis, Matijasevič and Robinson [1]:

(*) Does there exist an algorithm to determine if a sequence of formulas of types: $\alpha<\beta, \alpha \mid \beta, \alpha=\square$ has a solution in positive integers?

Received by the editors June 8, 1978 and, in revised form, July 10, 1978.

AMS (MOS) subject classifications (1970). Primary 10N05, $10 \mathrm{~B} 99$.

Key words and phrases. Diophantine problems, decidability.

${ }^{1}$ The author gratefully acknowledges the invaluable assistance of Professor M. Davis. Thanks are also due to David Bassain for his numerous helpful suggestions.

(c) 1979 American Mathematical Society 0002-9939/79/0000-0555/\$02.25 
This paper includes a general theorem concerning binary relations which has as a corollary an affirmative solution to (*).

In this paper $\alpha, \beta$ are either variables or positive integers and $N$ is the set of positive integers. $\alpha R \beta$ means " $\alpha$ is related to $\beta$ in relation $R$ ”,

$$
R(\alpha)=\{\beta \mid \alpha R \beta\}, \quad R(S)=\bigcup_{\alpha \in S} R(\alpha)
$$

Below, contexts will be encountered in which specific variables have their range restricted by stipulations already made. In such contexts, $\langle\alpha\rangle$ represents the set of possible values of the variable $\alpha$.

Definition. The family of computable functions $\left\{g^{(l)}: l \in N\right\}$ is called a generalized common multiple in the relation $R$ if for any $\alpha_{1}, \ldots, \alpha_{l}$,

$$
g^{(l)}\left(\alpha_{1}, \ldots, \alpha_{l}\right) \in \bigcap_{i=1}^{l} R\left(\alpha_{i}\right) .
$$

Consider computable relations $R_{1}, \ldots, R_{m}$ and computable sets $S_{1}, \ldots, S_{n}$. Let

$$
R_{U}=\bigcup_{i=1}^{m} R_{i}, \quad R_{I}=\bigcap_{i=1}^{m} R_{i}, \quad S_{I}=\bigcap_{i=1}^{n} S_{i},
$$

$P_{I}(\alpha)=R_{I}(\alpha) \cap S_{I}$. Then we have:

MAIN Theorem. If (i) for all $i=1, \ldots, m, \alpha R_{i} \beta \Rightarrow \alpha<f(\beta)$ for some strictly increasing computable function $f$,

(ii) there is a family $\left\{g^{(l)}: l \in N\right\}$ which is a generalized common multiple in the relation $P_{I}$.

For all $i=1,2, \ldots, m$ there exists $c_{0}$ such that

(iii) either for all $\alpha>c_{0} \alpha R_{i} \alpha$, in which case $R_{i}$ is called $c_{0}$-reflexive, or for all $\alpha>c_{0} \sim \alpha R_{i} \alpha$ in which case $R_{i}$ is called $c_{0}$-antireflexive, and

(iv) for all $\alpha>c_{0}, R_{U}\left(R_{U}(\alpha)-\{\alpha\}\right) \subseteq R_{U}(\alpha)-\{\alpha\}$,

then there exists an algorithm to determine whether or not a given system of conditions of the types $\alpha R_{i} \beta, \alpha \in S_{i}$, has a solution in positive integers.

Let $P$ be some given system consisting of $p_{0}$ conditions. The proof of the Main Theorem will use the following definitions, of which the second is inductive.

Definition. $L_{P}$ is the set of (numbers or variables) $\alpha$ such that $P$ contains a sequence of conditions of the form:

$$
\alpha R_{i_{1}} \alpha_{1}, \quad \alpha_{1} R_{i_{2}} \alpha_{2}, \quad \cdots, \quad \alpha_{w-1} R_{i_{w}} \alpha
$$

where some $R_{i, j}$ is $c_{0}$-antireflexive.

DEFINITION.

$B_{P}=\{c \in N:(\alpha R c) \in P\} \cup\left\{\alpha:(\alpha R \beta) \in P \wedge \beta \in\left(L_{P} \cup B_{P}\right)\right\}$.

Thus, $L_{P} \subseteq B_{P}$.

Let $c_{1}=\max _{c \in B_{P}} c, c_{2}=f^{\left(2 p_{0}\right)}\left(c_{0}\right)+f^{\left(p_{0}\right)}\left(c_{1}\right)$ where $f$ and $c_{0}$ are from the 
statement of the theorem, and $f^{\left(p_{0}\right)}$ is the function obtained by $p_{0}$ iterations of $f:$

Definition. $\bar{P}=\left\{\alpha R \beta:(\alpha R \beta) \in P \wedge \alpha, \beta \in B_{P}\right\} \cup\left\{\alpha \in S_{i}:(\alpha \in\right.$ $\left.\left.S_{i}\right) \in P \wedge \alpha \in B_{P}\right\}$.

The proof of the Main Theorem follows from the following.

LEMMA. $P$ has a solution in positive integers if and only if $\bar{P}$ has a solution in positive integers $<c_{2}$.

Proof. Suppose $P$ has a solution. Let $\alpha \in L_{P}$. Then there is the sequence of conditions (1) where $R_{i_{j}}$ is $c_{0}$-antireflexive. Now, suppose that $P$ had a solution in which $\alpha_{j-1}$ had a value $x>c_{0}$. Then $x \notin\left\langle\alpha_{j}\right\rangle$. Therefore, $\left\langle\alpha_{j}\right\rangle \subseteq$ $R_{u}(x)-\{x\}$, and

$$
x \in R^{(w-1)}\left(\left\langle\alpha_{j}\right\rangle\right) \subseteq R_{u}(x)-\{x\},
$$

using (1) and (iv). This contradiction shows that for any solution, $\alpha_{j-1} \leqslant c_{0}$ and $\alpha_{i} \leqslant f^{\left(p_{0}\right)}\left(c_{0}\right)$ for $i=1,2, \ldots, w$. Hence any solution of $P$ is such that all variables in $L_{P}$ have values $\leqslant f^{\left(p_{0}\right)}\left(c_{0}\right)$.

Next let $\alpha \in B_{P}$. Then $P$ contains a sequence of the form

$$
\alpha R_{i_{0}} \alpha_{1}, \quad \alpha_{1} R_{i_{1}} \alpha_{2}, \ldots, \quad \alpha_{w} R_{i_{w}} \beta
$$

where $w<p_{0}$ and $\beta$ is either a constant $\leqslant c_{1}$ or a variable in $L_{P}$ and hence with value $<f^{\left(p_{0}\right)}\left(c_{0}\right)$ in any solution. Then, in any solution of $P$,

$$
\begin{aligned}
\alpha & \leqslant f^{(w)}\left(\max \left(c_{1}, f^{\left(p_{0}\right)}\left(c_{0}\right)\right)\right) \\
& =\max \left(f^{(w)}\left(c_{1}\right), \dot{f}^{\left(w+p_{0}\right)}\left(c_{0}\right)\right)<c_{2} .
\end{aligned}
$$

Since all variables in $B_{P}$ have values $<c_{2}$ in any solution of $P, \bar{P}$ has such a solution.

Conversely, suppose $\bar{P}$ has such a solution. Then any "loop" of the form (1) in which no $R_{i_{j}}$ is $c_{0}$-antireflexive can be satisfied by

$$
\alpha=\alpha_{1}=\alpha_{2}=\cdots=\alpha_{w}>c_{0} .
$$

Consequently all such "loops" in $P$ can be eliminated by replacing each occurrence of $\alpha_{i}(i=1,2, \ldots, w)$ by $\alpha$. (But the value to be assigned $\alpha$ must be $>c_{0}$.)

For the purpose of this proof $\alpha$ is called a parent of $\beta$ if the condition $\alpha R_{i} \beta$ is in $P$ for some $i$, and the generation of $\alpha$, for $\alpha \notin B_{P}$, is the largest $w$ such that $P$ contains a sequence

$$
\alpha_{1} R_{i_{1}} \alpha_{2}, \quad \alpha_{2} R_{i_{2}} \alpha_{3}, \quad \ldots, \quad \alpha_{w} R_{i_{w}} \alpha
$$

where $\alpha_{2} \notin B_{P}$. Since $\bar{P}$ has a solution we can assign values to all $\alpha \in B_{P}$. Also assign all parentless $\alpha$ 's the value $s_{0}$ where $s_{0}=\min \left(S_{I}\right)$. At this point any variable of the first generation, say $\alpha$, has parents which are either constants or have already been assigned values. Suppose these parents have values $a_{1}, \ldots, a_{l}$. Then fix the value of $\alpha$ as $g^{(l+1)}\left(a_{1}, \ldots, a_{l}, f\left(c_{0}\right)\right) .\left(f\left(c_{0}\right)\right.$ is included as an argument in order to guarantee, by (i) that variables arising 
from collapsed "loops" are given values $>c_{0}$.) In this way the whole first generation is assigned values. Now any variable of the second generation has parents with definite values. Continue this process until all variables have been assigned values. These values constitute a solution of $P$ in positive integers.

Using the same notation as above and sacrificing some generality a much simpler statement of the Main Theorem can be obtained.

COROLlaRY. If (i) there is a family $\left\{g^{(l)}: l \in N\right\}$ which is a generalized common multiple in the relation $P_{I}$,

(ii) $R_{i}$ is reflexive or antireflexive, and

(iii) $\alpha R_{i} \beta$ implies $\alpha<\beta$,

then there is an algorithm to determine whether or not a system of conditions of types $\alpha R_{i} \beta, \alpha \in S_{i}$, has a solution in positive integers.

There are two interesting applications of this corollary.

The first gives a positive solution to the problem posed in [1].

COROLlARY. There is an algorithm to determine whether or not a system of conditions of types $\alpha<\beta, \alpha \mid \beta, \alpha=\square$ has a solution in positive integers.

The second finds a fine boundary line between decidable and undecidable problems.

THEOREM. There is an algorithm to determine whether or not a system of conditions of type $f_{i}(\beta)<\alpha$, where the $f_{i}$ are any recursive, strictly increasing functions, has a solution in positive integers. However for general nondecreasing functions $f_{i}$ there is no such algorithm.

Proof. The first assertion is an immediate consequence of the first corollary above where the $R_{i}$ of the corollary are $f_{i}(\beta)<\alpha$. To prove the second assertion, suppose there were such an algorithm. Then in particular there is an algorithm to determine whether or not there is a solution in positive integers to the system:

$$
f_{1}(\beta)-1<\alpha, \quad f_{1}^{-1}(\alpha)-1<\beta, \quad f_{2}(\gamma)-1<\alpha, \quad f_{2}^{-1}(\alpha)-1<\gamma
$$

where $f_{i}^{-1}(\alpha)=\min _{x}\left(f_{i}(x)>\alpha\right)$. (If the minimum does not exist let $f_{i}^{-1}(\alpha)$ be "infinite".) This sequence of formulas is equivalent to $f_{1}(\beta)=\alpha=f_{2}(\gamma)$. Consequently, if there were an algorithm to determine whether or not this system has a solution then there would be an algorithm to determine whether or not $\operatorname{Range}\left(f_{1}\right) \cap \operatorname{Range}\left(f_{2}\right)=\varnothing$. But since every computable set-and, in particular, every context-free set-is expressible as $\operatorname{Range}(f)$ for some increasing computable function $f$, we would then have an algorithm to determine whether $L\left(\Gamma_{1}\right) \cap L\left(\Gamma_{2}\right)=\varnothing$ where $\Gamma_{1}$ and $\Gamma_{2}$ are context-free grammars and where $L(\Gamma)$ is the language accepted by $\Gamma$. No such algorithm exists (cf., e.g., [2, p. 583]). 


\section{REFERENCES}

1. Martin Davis, Yuri Matijasevic and Julia Robinson, Hilbert's tenth problem. Diophantine equations: Positive aspects of a negative solution, Proc. Sympos. Pure Math., vol. 28, Amer. Math. Soc., Providence, R. I., 1976, pp. 323-378.

2. , Unsolvable problems, Handbook of Mathematical Logic, North-Holland, Amsterdam, 1977, pp. 567-594.

3. N. K. Kosovskii, On solutions of systems consisting of both word equations and word length inequalities, Zap. Naučn. Sem. Leningrad. Otdel. Mat. Inst. Steklov. (LOMI) 40 (1974), 24-29. (Russian)

4. Julia Robinson, Definability and decision problems in arithmetic, J. Symbolic Logic 14 (1949), 98-114.

5. Edward Schwartz, Existential definability in terms of some quadratic functions, Doctoral Dissertation, Yeshiva University, 1974.

6. Th. Skolem, Diophantische Gleichungen, Ergebnisse der Math. und ihrer Grenzgebiete, Band 5, Springer, Berlin, 1938.

Courant Institute of Mathematical Sciences, New York University, New York, New YoRK 10012 